\title{
Chaos based blood glucose noninvasive measurement: new concept and custom study
}

\author{
Li Cui ${ }^{1}$, Yuhang Dai ${ }^{2}$, Wenshi Li ${ }^{2, a}$ and Yejia Feng ${ }^{2}$ \\ ${ }^{1}$ China Tower, Suzhou Branch Company, 215011 Suzhou, China \\ ${ }^{2}$ Soochow University, Microelectronics Department, 215006 Suzhou, China
}

\begin{abstract}
Background. Non invasive monitoring of Blood Glucose (BG) has been a challenge calling for new accurate and fast measurement methods. Objective. To propose new concept of chaos based BG non invasive test aiming at personal customization requirements. Methods. First to build the compact RC model of tissue BG through impedance precision measuring Kit, then to simulate and soft-test BG by Boolean chaotic Codec circuits in soft tool Multisim 13.0, The third to capture the chaotic decoding outputs with the Kit plus PC in calculated signatures of resistor and phase of the tested impedance at the subjects' left wrist in synchronous test by Bayer BG meter. Results. All in controlled trials of Bayer BG meter, the chaotic BG modelling had gained three new compared formulae in merits of errors less than $1 \mathrm{mmol} / \mathrm{L}$ and latency less than 1 minute. Conclusion. During further verification of this chaotic test paradigm, the opened logic route of above methods will boost measurement experts' confidence in overcoming future problems of blood glucose monitoring in vivo.
\end{abstract}

\section{Introduction}

Four kinds of methods on Blood Glucose non invasive measurement are in chemistry, electrical, optical, and acoustic principles [1,2]. Till now there have been puzzles on non invasive measurement paradigm as multi variables effects. However the BG test accuracy and latency problems are two more prominent issues. So entering personalized customization era, how to understand easily creative application of classic biological impedance will undoubtedly open new window of old obstacles.

Chaos based Blood Glucose non invasive measurement built by the corresponding author was inspired by three events: (1) With white noise excitation to measure and compute BG in 2006 contributed by Master student Xiaobing Hou at Beijing University of Chinese Medicine; (2) Modulated +5 V DC-DC supply power in chaotic waves in auricular NIRS BCI in 2012 contributed by Brainchildren Lab of our research group; (3) Water conductivity test. Human body can be modelled into a warm water bag as embedded into a single branch voltage divider circuit under test $[3,4]$ !

Next we report the personalized customization journey of chaotic BG impedance test modelling.

Section 2 starts with digging out the RC model of BG tissue impedance (innovation point 1) $[5,6]$.

\footnotetext{
${ }^{a}$ Corresponding authors: lwshi@suda.edu.cn

This work was supported by the Natural Science Foundation of Jiangsu Province, China in 2014 (No. BK20141196). This work was supported by the 2015 Annual General College Graduate Research and Innovation Programs (No. KYLX15_1244) in Jiangsu Province, China.
} 
Section 3 focuses on RC-based chaotic Codec BG sensing (innovation point 2) [7,8].

Section 4 extracts three custom formulae in contrast test of Bayer BG meter (innovation point 3 ).

Section 5 throws up the conclusions caring the illustration of test accuracy and latency indexes.

\section{Wrist blood glucose modelling in RC net}

The idea searches for BG model in RC net. Figure 1 and 2 show needed instruments. Wherein the chip AD5933 is high speed biological impedance acquisition system in key parameters of $12 \mathrm{bit}$, 1MSPS.
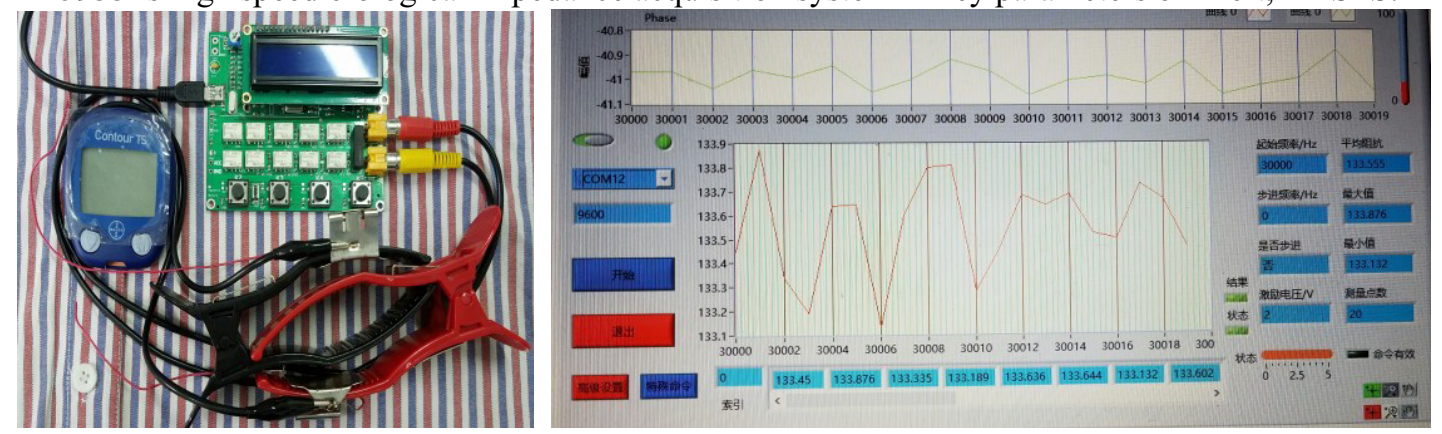

Figure 1. Bayer BG meter and Impedance precision measuring Kit (left) and PC terminal Interface (right).
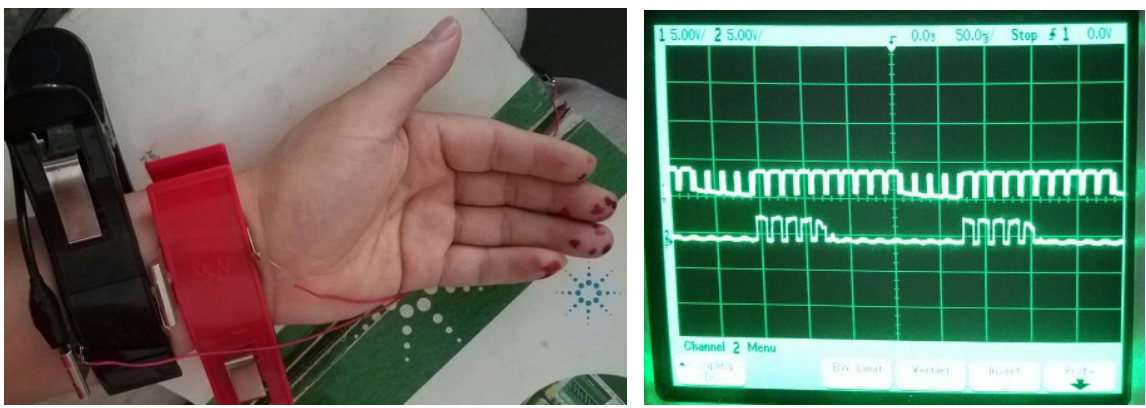

Figure 2. IEC standard ECG clamp and test site (left) and Agilent 54264 A Oscilloscope display of chaos (right).

Table 1. Compact RC model of tissue BG

\begin{tabular}{|c|c|}
\hline Tested BG / $\mathbf{~ m m o l} / \mathbf{L}$ & $\mathbf{R} / \mathbf{\Omega} / / \mathbf{C} / \mathbf{n F}$ \\
\hline 6.6 & $102 / / 11$ \\
\hline 7.3 & $104 / / 15$ \\
\hline 7.7 & $104 / / 33$ \\
\hline 8.8 & $104 / / 5$ \\
\hline 7.3 & $106 / / 328$ \\
\hline 7.6 & $106 / / 510$ \\
\hline
\end{tabular}




\begin{tabular}{|c|c|}
\hline 6.8 & $105 / / 75$ \\
\hline 6.7 & $109 / / 78$ \\
\hline
\end{tabular}

The accuracy of Bayer BG meter is less than $0.6 \mathrm{mmol} / \mathrm{L}$ with test time 8 of seconds.

Subjective: Male, 52 years old in healthy state@ room temperature of 28 degrees Celsius, Humidity of $60 \%$.

Step-1: BG test value sampling invasively after Alcohol disinfection and filling Tab. 1.

Step-2: During 5 minutes interval, to uniformly capture 5- 10-group data (20-trial per group set in one second). To anti-compute the RC model from the wrist impedances and then fill Tab. 1.

Step-3: To use the RC model in Table 1 as the "wrist in vivo" under test by Multisim 13.0 exploring new chaotic Codec BG test method.

\section{RC-based chaotic Codec BG sensing}

Figure 3 introduces this section's idea flowchart in detail. Boolean chaotic circuit is with coding core of XOR gate (U2A) and with decoding core of XOR gate (U5A), the known devided voltage branch includes the $\mathrm{RC}$ model $(\mathrm{R} 1 / / \mathrm{C} 1)$ and sampling resistor $\mathrm{R} 2$ and the chaotic decoding output load $\mathrm{R} 3$. The key waveforms (right) cares ports of IO1 IO4. Based on Table 1 we run 8 times for constructing Figure 4 (left) with the right mapping under the standard of Point $5(7.3 \mathrm{mmol} / \mathrm{L})$.

Summary: The new formula $\mathrm{y}=f(\mathrm{x})($ Equation 0$)$ is with error less than $1 \mathrm{mmol} / \mathrm{L}$.
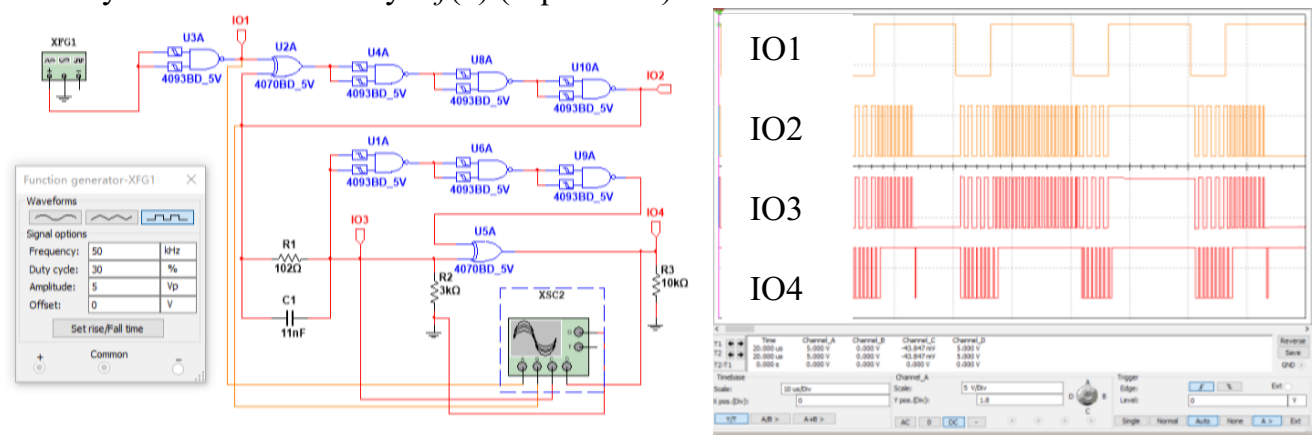

Figure 3. RC model based new chaotic coding and decoding BG soft-test (left) and key waveforms (right).
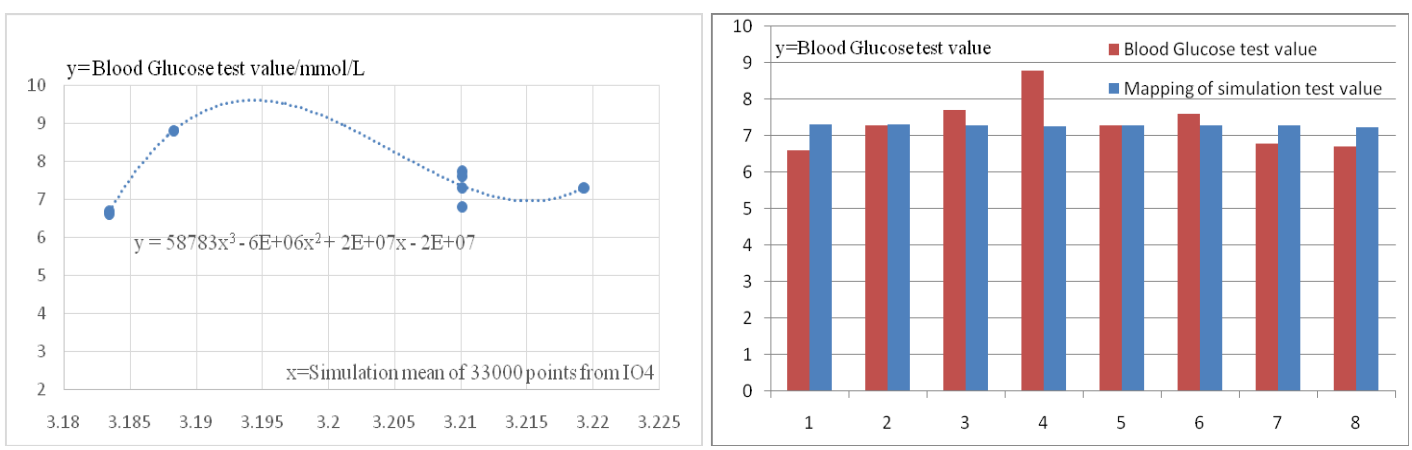

Figure 4. Soft-test of BG with simulation data (left) and their mapping "true" values (right). 


\section{Custom Chaos BG glucose non-invasive monitoring}

In fact like in Figure 3 we set generator frequency of $50 \mathrm{kHz}, 30$ percents of Duty in chaotic coding block with MCU of MSP430G2553. And the acquisition methods are also kept the same style in Section 2.

Figure $5 \sim$ Figure7 illustrate the mappings of wrist BG Resistors and Phases respectively with three kinds of extracting mean constrains: of 200R (P), of 30\% largest ones and of $30 \%$ smallest ones.
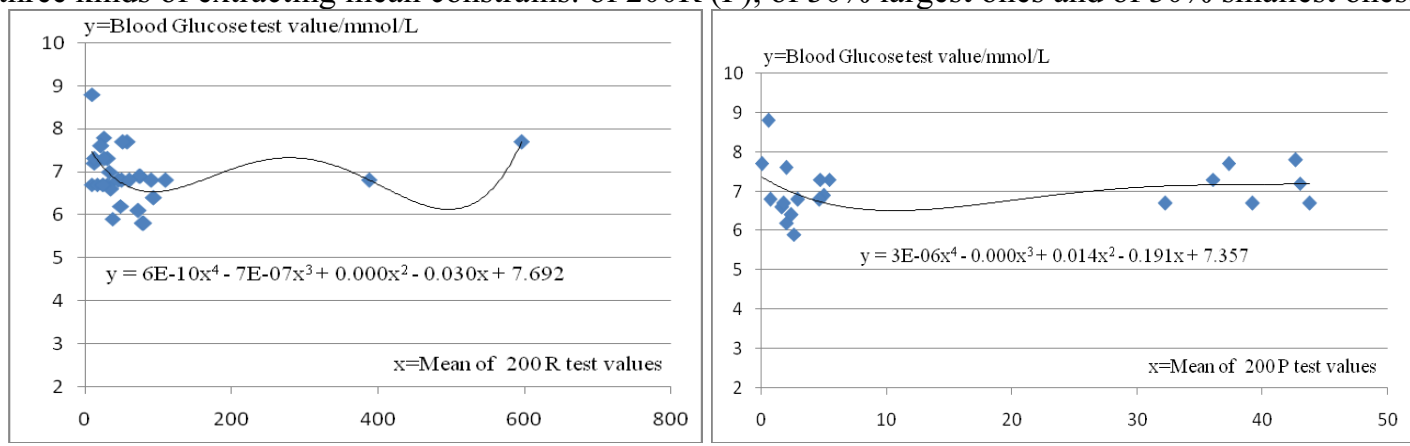

Figure 5. Chaotic impedance test based BG mapping (left R: mean) and (right P: mean).
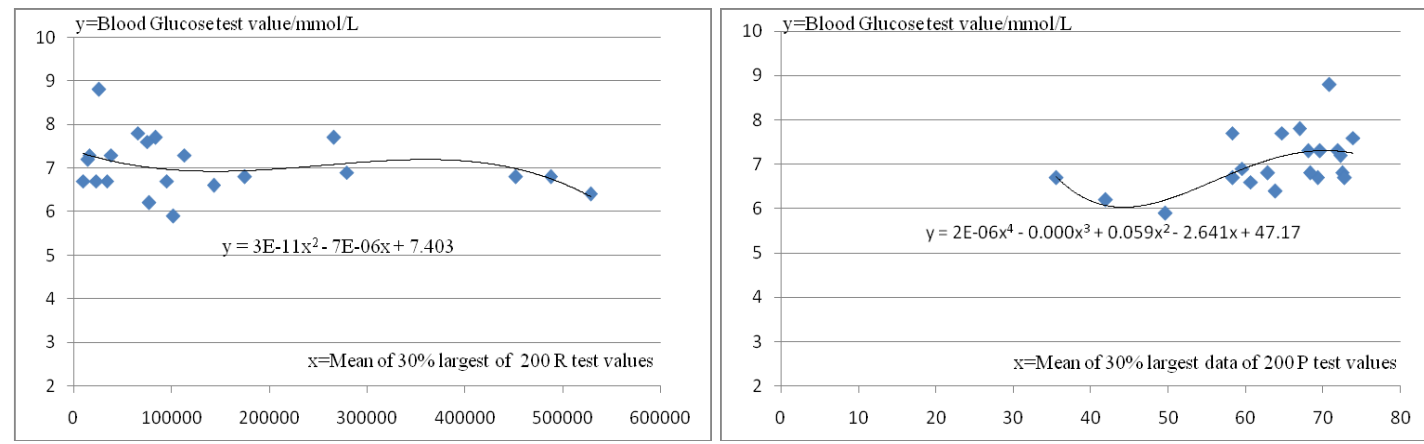

Figure 6. Chaotic impedance test based BG mapping (left R: mean of 30\% Max) and (right P: mean of 30\% Max).
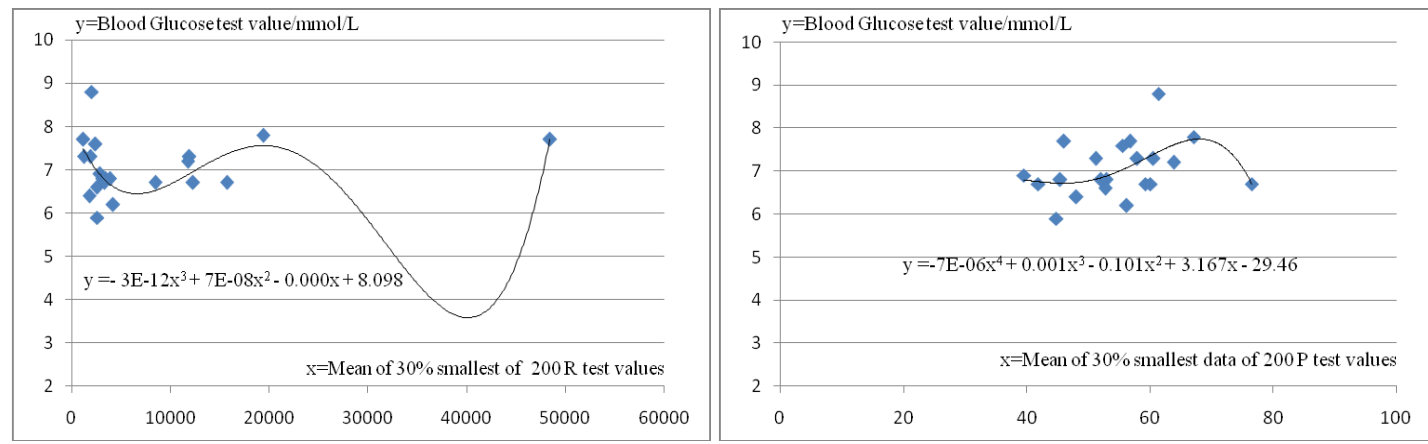

Figure 7. Chaotic impedance test based BG mapping (left R: mean of $30 \%$ Min) and (right P: mean of $30 \%$ Min). 


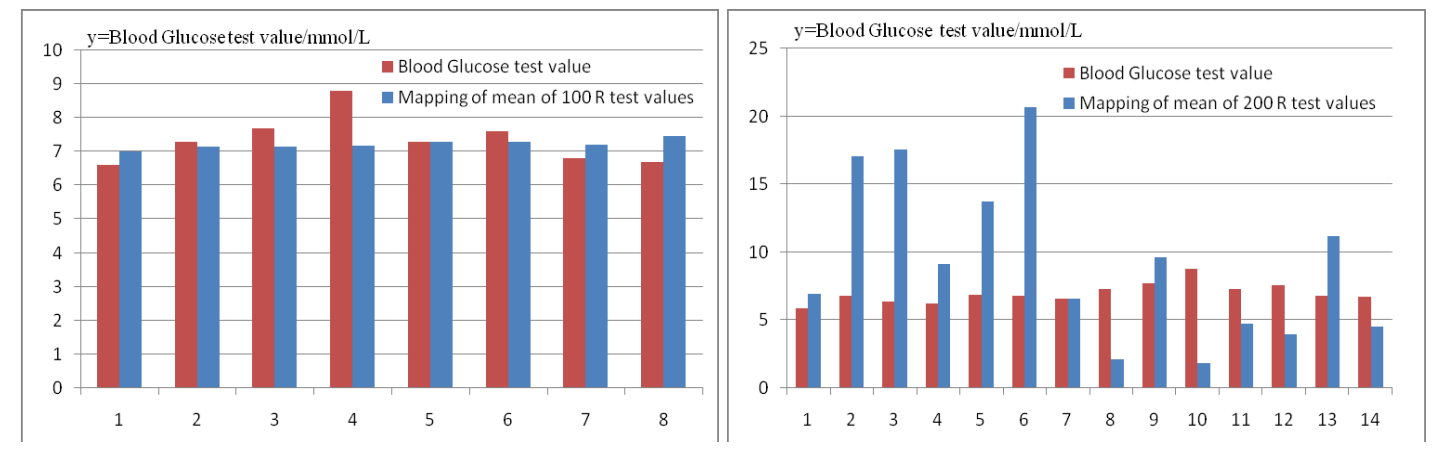

Figure 8. Impedance test based BG mapping (left: Sine wave excitation) and (right: Chaotic Codec)

To analyze Figure $5 \sim$ Figure 7 we must point out that all the derailed dots are for reason of $30 \mathrm{kHz}$ stimulus in Kit in Fig.1 (All data is met before and after the continues four lunches).

To compare Figure $8 @$ left and right we believe that classic inputs will result in basic accuracy of BG test mapping while in about 5 minutes delay times, fortunately the right results in Figure 8 with all $50 \mathrm{kHz}$ in Kit and in Codec circuits met minute-level latency.

\section{Conclusions}

To help build new RC model of BG non invasive measurement our co-works had shown the personalized customization method from test to simulation to test all in contract of Bayer BG meter monitoring for Subject One.

The grasped optimized experiments' condition includes 30\% Duty $40 \mathrm{kHz} \sim 50 \mathrm{kHz}$ excitation, and the digital Boolean Codec tech with $1 \sim 3$ stages delay chain@ room temperature of 28 degrees Celsius, Humidity of $60 \%$.

Further works will inhibit the effect from the temperature variable in order to stabilize this accuracy of about $0.5 \mathrm{mmol} / \mathrm{L}$ in chaotic measurement for Blood Glucose monitoring in vivo.

\section{References}

1. J. Yadava, A. Rania, V. Singha, Biomedical Signal Processing and Control, 18 (2015).

2. M. Goodarzi, S. Sharma, H. Ramon, Trends in Analytical Chemistry, 67 (2015).

3. Li Wenshi, Chinese Journal of Electronics, 25 (2016).

4. R. Brown, L. O. Chua, B. Popp, Int. J. of Bifurcation and Chaos, 2 (1992).

5. A. Caduff, E. Hirt, Yu Feldman, Biosensors and Bioelectronics, 19 (2003).

6. K. Song, U. Ha, S. Park, J. Bae, Hoi-Jun Yoo, IEEE J. of Solid-State Circuits, 50 (2015).

7. B. Muthuswamy, L. O. Chua, Int. J. of Bifurcation and Chaos, 20 (2010).

8. Rui Zhang. Physical Review E, 80 (2009). 